\title{
Cognitive workload and driving behavior in persons with hearing loss
}

\author{
Birgitta Thorslund, Björn Peters, Björn Lidestam and Björn Lyxell
}

\section{Linköping University Post Print}

\section{Tweet}

N.B.: When citing this work, cite the original article.

Original Publication:

Birgitta Thorslund, Björn Peters, Björn Lidestam and Björn Lyxell, Cognitive workload and driving behavior in persons with hearing loss, 2013, Transportation Research Part F: Traffic Psychology and Behaviour, (21), 113-121.

http://dx.doi.org/10.1016/j.trf.2013.09.011

Copyright: Elsevier http://www.elsevier.com/

Postprint available at: Linköping University Electronic Press

http://urn.kb.se/resolve?urn=urn:nbn:se:liu:diva-101198 


\title{
Cognitive workload and driving behavior in persons with hearing loss
}

\author{
Birgitta Thorslund $^{1,2}$, Björn Peters ${ }^{1,2}$, Björn Lidestam ${ }^{2}$, Björn Lyxell ${ }^{2}$ \\ ${ }^{1}$ VTI (Swedish National Road and Transport Research Institute) \\ ${ }^{2}$ Linnaeus Centre HEAD, Linköping University \\ Linköping, Sweden
}

Contact: BIRGITTA.THORSLUND@ VTI.SE

Purpose: To compare the effect of cognitive workload in individuals with and without hearing loss, respectively, in driving situations with varying degree of complexity.

Methods: 24 participants with moderate hearing loss (HL) and 24 with normal hearing (NH) experienced three different driving conditions: Baseline driving; Critical events with a need to act fast; and a Parked car event with the possibility to adapt the workload to the situation. Additionally, a Secondary task (observation and recalling of 4 visually displayed letters) was present during the drive, with two levels of difficulty in terms of load on the phonological loop. A tactile signal, presented by means of a vibration in the seat, was used to announce the Secondary task and thereby simultaneously evaluated in terms of effectiveness when calling for driver attention. Objective driver behavior measures ( $M$ and $S D$ of driving speed, $M$ and $S D$ of lateral position, time to line crossing) were accompanied by subjective ratings during and after the test drive.

Results: HL had no effect on driving behavior at Baseline driving, where no events occurred. Both during Secondary task and at the Parked car event HL was associated with decreased mean driving speed compared to baseline driving. The effect of HL on the Secondary task performance, both at Baseline driving and at the lower Difficulty Level at Critical events, was more skipped letters and fewer correctly recalled letters. At Critical events, task difficulty affected participants with HL more. Participants were generally positive to use vibrations in the seat as a means for announcing the Secondary task.

Conclusions: Differences in terms of driving behavior and task performance related to HL appear when the driving complexity exceeds Baseline driving either in the driving task, Secondary task or a combination of both. This leads to a more cautious driving behavior with a decreased mean driving speed and less focus on the Secondary task, which could be a way of compensating for the increasing driving complexity. Seat vibration was found to be a feasible way to alert drivers with or without HL. 


\section{Introduction}

Driving a car is one of the most complex and safety critical everyday tasks in modern society (Groeger, 2000). The Task Capability Interface (Fuller, 2000) describes driver control as when the demands of the driving task are less than the capability of the driver. Consequently, loss of control arises when the demands of the driving task exceed the driver's capability. The level of difficulty in the driving task is thus the inverse of the spare capacity and also connected to the cognitive workload (Fuller, 2005). Driving effort is dynamic as the cognitive demands can change back and forth from very low to extremely high, sometimes within fractions of a second (Michon, 1985; Peters, 2006). Among the factors determining the driving task demand, of which the driver has immediate and direct control, driving speed is the most significant (Fuller, 2005). It has been demonstrated that when a threshold of a certain preferred driving speed is exceeded, experienced task difficulty, effort and feeling of risk is affected (Lewis-Evans, 2011). Normal driving can be considered as a cognitive and controlled task, and thus one approach to understand driving behavior is using cognitive psychology. This approach attempt to describe internal processes involved in making sense of the environment, and deciding what action might be appropriate (Eysenck \& Keane, 2010; Neisser, 1976). Hearing loss (HL) means loss of auditory information, which may affect road user behavior in specific traffic situations and might reduce traffic safety (Ivers, Mitchell, \& Cumming, 1999; Schmolz, 1987).

The working memory plays a central role in processing of information. The phonological loop is one component in Baddeley's multicomponent model of the working memory (A. Baddeley, D., 2012; Repovs \& Baddeley, 2006). Within this loop, a phonological store holds memory traces in phonological form, and an articulatory rehearsal process recodes information from other modalities (A. D. Baddeley, 1983; Repovs \& Baddeley, 2006). Andersson (2002) demonstrated that specific aspects of the phonological system deteriorate as a function of poor auditory stimulation in individuals with HL. Specifically, the phonological representations are deteriorating and this deterioration also affects the ability to rapidly perform phonological operations (i.e., analyze and compare letters) (Andersson, 2002). Thus, it is reasonable to assume that a Secondary task during driving, which includes performing phonological operations would affect drivers with HL more than normal hearing (NH) drivers.

The prevalence of age-related HL is increasing, due to populations becoming progressively older and thus presenting symptoms of reduced sensory function. These may be physiological or caused by additional factors damaging the hearing function (e.g., harmful noise in the 
environment). The prevalence increases for all ages, although the most common category of HL is presbycusis, which refers to the physiological age-related changes of the peripheral and central auditory system leading to HL. The prevalence of HL in Europe is roughly $30 \%$ for men and $20 \%$ for women at the age of 70 years, and $55 \%$ for men and $45 \%$ for women at the age of 80 years (Roth, 2001). A consequence of the increasing prevalence of HL, is that the number of road users (not only drivers) with HL will also increase.

Relatively few studies have focused on HL and driving previously, and the knowledge is thus rather limited. However, the importance of a hearing capability for road users has been examined by Schmolz (1987), who revealed that HL is associated with higher degree of inattention and that traffic education for older adults should be bound to hearing ability. There are also some studies with either self-reported accidents or medical record data, pointing to connections between HL and higher risks of traffic accidents (Ivers, et al., 1999; McCloskey, Koepsell, Wolf, \& Buchner, 1994; Picard, et al., 2008).

Thorslund, Peters, Lyxell and Lidestam (2013) showed that among individuals with HL there are fewer license holders and that the prevalence of driving license decreases with an increasing degree of HL. However, those drivers who have HL drive to the same extent as NH drivers (Thorslund et al., 2013). The results also revealed that drivers with HL may develop compensatory strategies, since the number of incidents or accidents is not related to HL (Thorslund, et al.). The interest in a warning system for inattention was high among all participants regardless of HL or not (Thorslund, et al.). This further stresses the importance of research on drivers with $\mathrm{HL}$, to examine driving behavior and possible compensatory strategies, and also on feasible warning modalities and design of support systems accessible for drivers with HL.

Due to a more complex in-vehicle environment of systems for infotainment (e.g., navigation system, mobile phone), there is an increased risk of distracting the driver from the driving task. Hickson, Wood, Chaparro, Lacherez, and Marszalek (2010) reported a significant interaction between HL and distractors, such that people with moderate to severe HL had significantly poorer driving performance in the presence of distractors than had individuals with NH or mild HL. This further supports that it is important to design driver support systems also for drivers with HL.

According to Fuller (2005), the driver selects a range of workload and then drives in such a way as to maintain the experienced difficulty within that range. Manipulation of driving speed along with degree of engagement in Secondary tasks is claimed to be the primary mechanisms 
for this maintenance (Fuller, 2005). Simulator studies have demonstrated that distraction tasks (both visual and cognitive) lead to decreased speed and a difficulty in maintaining a certain speed (Engström, 2005; Lewis-Evans, 2011). Tsimhoni (2003) found, in a driving simulator study with a Secondary task and varying complexity of the road condition, that the total time allocated to the Secondary task increased significantly with a more complex driving task (sharp curves vs. straight road vs. parking). Even though the participants seemed to adapt their glance behavior to the road conditions, driving performance still deteriorated with a more complex environment (more lane departures), and at the same time more errors were made in the in-vehicle task (Tsimhoni, 2003).

The purpose of the present simulator study was to compare the effect of cognitive workload, in individuals with and without HL, respectively, in driving situations with various complexities. We specifically examined, during three different driving conditions, two levels of difficulty in terms of load on the phonological loop. A tactile signal, provided as a vibration in the seat, was evaluated for effectiveness when calling for driver attention. A comparison was made between drivers with moderate HL and drivers with NH. All participants experienced Baseline driving with low cognitive effort, Critical events with a need to act fast, and a Parked car event with the possibility to adapt the workload to the situation (e.g., give less priority to the Secondary task). The Secondary task was present at all driving conditions.

\section{Method}

\section{Participants}

The participants ( $N=48 ; n=24$ in the NH group, $n=24$ in the HL group) were recruited from the region around Linköping. The NH group included 12 men and 12 women and the HL group included 13 men and 11 women. On average the NH group drove $1892 \mathrm{~km} /$ year $(S D=$ $1095 \mathrm{~km} /$ year) and the HL group drove $1545 \mathrm{~km} /$ year $(S D=673 \mathrm{~km} /$ year $)$. The mean age was 60.1 years $(S D=7.1$ years) for NH men, $M=59.6$ years $(S D=5.0$ years) for NH women, $M=$ 62.0 years $(S D=7.9$ years) for HL men and $M=61.0$ years ( $S D=9.8$ years) for HL women. The inclusion criterion for the NH group was a hearing threshold of maximum $20 \mathrm{~dB}$ at each frequency $(500,1000,2000$ and $4000 \mathrm{~Hz})$ measured with a pure tone audiometer. Inclusion criterion for the HL group was a moderate HL (41-70 dB) according to WHO categories (Arlinger, 2007) measured with a pure tone average of four mean values, PTA4 (mean of 500, 1000, 2000 and $4000 \mathrm{~Hz}$ ). Participants with HL were asked to use their hearing aids when 
driving if they normally do and $16(67 \%)$ did. Table 1 displays audiogram data for the men and women, respectively, in the HL group.

Table 1 about here

\section{Procedure}

The driving scenario was a $35 \mathrm{~km}$ long rural road with a speed limit of $70 \mathrm{~km} / \mathrm{h}$. The traffic density was low with no traffic in the same lane and approaching cars approximately every 22 seconds. The Baseline driving consists of the sequences during this drive where no critical events or Parked car event were present.

For the Critical events, to create near collisions, the drivers were distracted by means of the Secondary task (reading and repeating letters from a screen placed at a relative large downward angle (40-45 degrees), and then "pushed" across the median towards an oncoming vehicle by introducing a steering angle in the simulated vehicle without submitting this information to the motion platform.

The Parked car event was a situation when the participants saw a parked car ahead (from 360 meters) with warning lights activated and they were prompted to perform the Secondary task at a distance of 70 meters from the parked car.

During baseline driving and at critical events, all participants experienced two different Difficulty Levels of the Secondary task. At the Parked car event, all participants experienced the more demanding level. Twice per minute drivers were prompted by a vibration in the seat to first look at and then read back a complete sequence of four letters appearing on the display, one at a time. To create two levels of cognitive workload, the phonological similarity effect was used (Conrad \& Hull, 1964) and the sequences consisted of randomized letters that were either phonological alike (e.g., BDPT) or not phonological alike (e.g., RKNJ), to create two Difficulty Levels. Each letter was displayed for $0.7 \mathrm{~s}$, with no pause between letters, creating total stimulus duration of 2.8 s. This is an adaptation from Sternberg's scanning paradigm (Sternberg, 1966), where a set of 1 to 6 digits were presented sequentially to the subject at the rate of a digit every $1.2 \mathrm{~s}$, which was pragmatically tested. The display time had to be long enough for recognition of the letters, but short enough for the participants to keep their eyes on the display. The total duration of the task corresponds to the necessity of creating a critical situation when the drivers take the eyes off the road to look at the display. The instruction was to look at all four letters and then repeat the whole sequence in the correct order. 


\section{Performance indicators and measures}

Driver behavior measures were Mean Driving speed; Standard deviation (SD) Driving speed; Mean Lateral position (LP); Standard deviation Lateral position (SDLP); and Minimum Time to line crossing (TLCmin) (Brookhuis, 2003). The objective measures were also accompanied by subjective ratings during and after the test drive. These aimed at evaluating the realism of the simulated event. For subjective ratings during the test drive, the following question was presented on the screen after each event: How critical did you experience the situation to be? The participants answered on a scale from $1=$ not critical at all to $7=$ extremely critical. This is an adaptation (specifically the dimension of the degree of complication) from the Situation Awareness Rating Technique (SART) 10 Dimension-scale, each dimension ranging from 1 to 7, which is one of the best known and widely used scales to assess situation awareness (Endsley \& Garland, 2000). For subjective ratings after the test drive, a questionnaire with questions answered on 5-point scales, where 1 was "not at all" and 5 was "very much" (e.g., how realistic did you experience the simulator to be?) were answered. Eye tracking was used as a quality measure of performed task. However, the results from the eye tracking data will be presented in a separate paper.

\section{Design and expectations}

A mixed $2 \times 2 \times 2$ factor design with the fixed factors Hearing Status (NH vs. HL), Gender (men vs. women), and Difficulty Level (lower vs. higher, operationalized as phonologically similar vs. dissimilar letters in the Secondary task), and Participant (participant 1-48) within Hearing Status as a random factor was used. Main effects of Hearing Status, Gender and Difficulty Level as well as interaction effects (Hearing Status $\times$ Gender, Hearing Status $\times$ Difficulty Level, Hearing Status $\times$ Gender $\times$ Difficulty Level) were examined for all performance indicators and measures. Driving behavior measures included three different levels: Baseline driving; Secondary task; and Parked car event. Two Difficulty Levels of Secondary task were experienced both during Baseline driving and during Critical events. During the Parked car event only the more difficult level of Secondary task was experienced, see Table 2.

Table 2 about here

Three main assumptions were examined. Firstly, participants with HL were expected to show a more cautious driving behavior. This means that for the HL group, compared to the $\mathrm{NH}$ group, the Driving speed would be lower, Safety margins would be larger, and the motivation 
to perform the Secondary task would be lower, resulting in lower Secondary task performance.

Secondly, the effect of increasing complexity in the driving situations was assumed to be larger on the HL group, compared to the $\mathrm{NH}$ group, meaning the Brake distance before the parked car would be longer for the HL group, the driving speed around the Parked car event would be lower, and the Secondary task performance would decrease more.

Thirdly, the phonological similarity effect was expected to strike harder on Participants with HL. This means that for the HL group, compared to the NH group, there would be a more apparent effect of task difficulty on the Secondary task performance.

\section{Analysis}

Analysis, with planned comparisons within and between the Hearing Status levels, was carried out on the three driving conditions respectively, using a mixed linear model. In addition, the Secondary task performance at each driving condition respectively was analysed, also with planned comparisons in a mixed linear model. All effects reported as significant have an alpha level of $p<.05$ and are presented with an $F$-value and the effect size measure of partial eta squared, $\eta_{\mathrm{p}}{ }^{2}$. In the present study, with 48 participants, having a two-factor design with Gender and a Hearing Status, a two-tailed effect size of 0.8 , gives a power of approximately 0.77 . The effects size for $F(1,44)$ and $p<.05$ will be at least $\eta_{\mathrm{p}}{ }^{2}=.08$.

Driving behaviour measures during Baseline driving and at the Parked car event was examined in terms of Mean Driving Speed, SD Driving Speed, Mean LP, SDLP, and Mean TLCmin. Driving behaviour measures during critical events are not presented in this paper.

The Secondary task performance was analyzed for Baseline driving, Critical events and Parked car event, respectively. Dependent variables were Number of correct recalled letters per task, Number of skipped letters per task, and Number of correct recalled letters ignoring order. See Table 3 for examples of these measures. At baseline driving and the Parked car event, Hearing Status, Gender and the interaction of Hearing Status and Gender were included as fixed effects.

\section{Table 3 about here}

The number of Secondary tasks presented at baseline driving differed for each person, due to driving speed. Thus the Number of correct recalled letters per task, Number of skipped letters per task and Number of correct recalled letters ignoring order are presented as percentages. 
The subjective ratings (adapted SART and questionnaire) were analyzed with ordinal logistic regression and the results are presented with Odds Ratio $(O R)$ and significance interval of 95\% (CI95). OR $>1$ means higher rating and OR $<1$ means lower rating. Main effects of Gender and Hearing Status were examined as well as interaction effects.

\section{Results}

First the results of driving behavior measures from each driving task are presented: Baseline driving; Driving at Parked car event; and Driving during critical events. These are followed by results from the Secondary task at each driving task, respectively.

\section{Baseline driving}

During baseline driving, without Secondary task and thus only the driving task to focus on, there was no significant main effect of Hearing Status or Gender on Mean driving speed or on SD driving speed. Neither was there a significant interaction effect between Hearing Status and Gender on these Performance indicators.

No significant main effect of Gender or of Hearing Status emerged for Mean LP and there was no significant interaction effect between Hearing Status and Gender. Neither were there significant main effects or interaction effects of Hearing Status and Gender on SDLP. This means all participants drove relatively straight within the lane.

There was a significant main effect of Gender on Mean TLCmin, such that men had approximately $1 \mathrm{~s}$ longer Mean TLCmin (approx. $7 \mathrm{~s}$ vs. $6 \mathrm{~s}$ ), $F(1,44)=5.31, p=0.03, \eta_{\mathrm{p}}{ }^{2}=$ 0.11. There was also a significant interaction effect for Gender and Hearing Status, such that HL affected Mean TLCmin more for men than for women, $F(1,44)=4.60 p=0.04, \eta_{\mathrm{p}}{ }^{2}=$ 0.10. No significant main effect of Hearing Status emerged on Mean TLCmin.

The questionnaire after driving revealed that the self-rated driving performance was just above average $(M=3.31 ; S D=0.56$ on the scale from $1=$ very bad, to $5=$ very good $)$. There was no significant main effect of Gender or Hearing Status and neither was there an interaction effect of Gender and Hearing Status on the self-rated driving performance.

The general realism of the driving situation in the simulator was rated as high $(M=3.72, S D=$ 0.71 on the scale from $1=$ not at all realistic to $5=$ very realistic) and men experienced the simulator as significantly more realistic $(O R=17.1$, CI95 $=2.9-82.7)$. A significant effect of Hearing Status emerged, such that NH drivers experienced the simulator as more realistic (OR 
$=6.3$, CI95 $=1.1-37.5)$. There was no significant interaction effect between Hearing Status and Gender.

Regarding simulator sickness (rated on the scale from $1=$ experienced no sickness at all, to 5 = experienced very much sickness), significant interaction effects of Gender and Hearing Status was found. Females in the HL group experienced more simulator sickness compared to females in the $\mathrm{NH}$ group $(O R=14.4$, CI95 $=2.4-86.7)$ and females in the HL group experienced more simulator sickness than men in the HL group $(O R=14.3$, CI95 $=2.5-82.9)$. See Table 4 for mean values and standard deviations.

Table 4 about here

The realism of the simulator sound was rated slightly above average $(M=3.33, S D=0.99$ on the scale from $1=$ not realistic at all, to $5=$ very much realistic) and no significant effects emerged for Hearing Status or Gender.

Regarding realism of simulator vibration (rated on the scale from $1=$ not realistic at all, to $5=$ very realistic), a significant effect emerged within the HL group, such that women experienced less vibration realism than men did $(O R=0.18$, CI95 $=0.03-0.90)$.

To summarize, the differences related to HL during baseline driving in the simulator were few and mainly focused on the experience of the driving simulator. Drivers with NH experienced the simulator as more realistic. Female drivers with HL experienced the most motion sickness and had the lowest ratings of vibration realism. A significant result in the driving behavior measure is that the effect of HL on Mean TLCmin is larger for men than for women.

\section{Driving at Parked car event}

There was a significant main effect of Gender on the Mean driving speed when passing the parked car, such that men drove approximately $5 \mathrm{~km} / \mathrm{h}$ faster $F(1,44)=4.24, p=0,04, \eta_{\mathrm{p}}{ }^{2}=$ 0.09. There was also a significant main effect of Hearing Status on the Mean driving speed in this situation, such that participants with HL drove approximately $5 \mathrm{~km} / \mathrm{h}$ slower, $F(1,44)=$ 2.42, $p=0.05, \eta_{\mathrm{p}}{ }^{2}=0.05$. There was no significant interaction effect between Hearing Status and Gender on Mean driving speed. Mean driving speed and standard deviation for both Hearing Statuses respectively are displayed in Figure 1.

Figure 1 about here 
For Braking distance when approaching the parked car no significant effect emerged for Hearing Status or Gender. Neither was there an interaction effect between Gender and Hearing Status. All participants performed the Secondary task at this event.

In sum, both Hearing Status and Gender affected the driving speed when approaching and passing a parked car. Women drove slower and participants with HL drove slower during this event.

\section{Driving behavior during the Secondary task}

During baseline driving with the Secondary task, a significant main effect of Hearing Status emerged for Mean driving speed, such that participants with HL drove approximately $6 \mathrm{~km} / \mathrm{h}$ slower during Secondary task than NH participants did (approx. $65 \mathrm{~km} / \mathrm{h}$ vs. $70 \mathrm{~km} / \mathrm{h}$ ), $F(1$, $44)=7.68, p=0.01, \eta_{\mathrm{p}}{ }^{2}=0.14$. A significant main effect of Task difficulty arose, such that participants drove approximately $1 \mathrm{~km} / \mathrm{h}$ faster during the higher Difficulty Level, $F(1,44)=$ 15.42, $p=0.00, \eta_{\mathrm{p}}{ }^{2}=0.26$. No significant main effect of Gender emerged and no interaction effects turned out to be significant.

There was a significant main effect of Task difficulty on SD driving speed, such that variation in speed decreased with increasing task difficulty (approx. $6 \mathrm{~km} / \mathrm{h}$ vs. $9 \mathrm{~km} / \mathrm{h}$ ), $F(1,44)=$ 39.16, $p=0.00, \eta_{\mathrm{p}}{ }^{2}=0.47$. No significant main effect of Gender or Hearing Status was found. Neither were there significant interaction effects.

For Lateral position, a significant main effect of Gender emerged, such that women drove $0,10 \mathrm{~m}$ closer to the left, $F(1,44)=5.38, p=0.02, \eta_{\mathrm{p}}{ }^{2}=0.10$. There was also a significant main effect of Task difficulty, such that participants drove $0.05 \mathrm{~m}$ closer to the left at the higher Difficulty Level $F(1,44)=43,32, p=0.00, \eta_{\mathrm{p}}{ }^{2}=0.50$. No significant main effect emerged for Hearing Status and there were no significant interaction effects.

There was a significant main effect of Task difficulty on SDLP, such that there was more variation in LP at the less demanding level than at the more demanding level $(0,26 \mathrm{~cm}$ vs. $0,19 \mathrm{~cm}), F(1,44)=98.50, p=0.00, \eta_{\mathrm{p}}{ }^{2}=0.69$. There was no significant main effect of Gender or Hearing Status and neither were there any interaction effects on SDLP.

For Mean TLCmin, a significant main effect of gender emerged, such that men had a longer Mean TLCmin (approx. 6 s vs. 5 s), $F(1,44)=6.06, p=0.02, \eta_{\mathrm{p}}{ }^{2}=0.10$. Also for Hearing Status a significant main effect emerged, such that $\mathrm{NH}$ participants had a longer Mean TLCmin than participants with HL had (approx. $6 \mathrm{~s}$ vs. $5 \mathrm{~s}$ ), $F(1,44)=2.85, p=0.05, \eta_{\mathrm{p}}{ }^{2}=$ 
0.08. There was a significant interaction effect for Gender and Hearing Status, such that HL affected Mean TLCmin more for men $F(1,44)=7.75, p=0.01, \eta_{\mathrm{p}}{ }^{2}=0.12$. There was no significant main effect of Task difficulty and no further interaction effects.

The questionnaire after driving showed that the participants were positive towards the way of announcing the Secondary task by seat vibration $(M=4.60, S D=0.68$ on the scale from $1=$ not feasible at all, to $5=$ very feasible). No significant effect of Hearing Status or Gender emerged. The difficulty of Secondary task was rated shortly above average $(M=3.3, S D=0.8$ on the scale from $1=$ very easy, to $5=$ very difficult). A significant main effect of Gender emerged, such that men experienced the Secondary task as significantly less difficult $(O R=$ 0.20, CI95 = 0.04-0.95). No significant main effect of Hearing Status emerged.

To summarize, the effect of HL on driving behavior during Secondary task includes decreased driving speed and, in line with baseline driving, decreased Mean TLCmin (especially for men with HL).

\section{Secondary task performance at baseline driving (2 levels)}

A significant main effect emerged for Hearing Status, such that $\mathrm{NH}$ participants had higher percentage correct, $F(1,44)=3.52, p=0.05, \eta_{\mathrm{p}}{ }^{2}=0.08$. There was no significant main effect of Gender and no effect of Task difficulty on percentage correct. Neither was there any interaction effects found.

For percentage skipped letters, a significant main effect emerged for Gender, such that women skipped more letters than men did, $F(1,44)=4.60, p=0.04, \eta_{\mathrm{p}}{ }^{2}=0.09$. There was also a significant main effect of Hearing Status, such that participants with HL skipped more letters than NH participants did, $F(1,44)=6.10, p=0.02, \eta_{\mathrm{p}}{ }^{2}=0.12$. There was no significant effect of Task difficulty on percentage skipped and no significant interaction effects were found.

The number of correct recalled letters, regardless of the order, showed a significant main effect for Hearing Status, such that participants with NH performed better than participants with HL, $F(1,44)=9.91, p<0.001, \eta_{\mathrm{p}}{ }^{2}=0.18$. There were neither significant effects of Gender or Task difficulty on percentage correct when ignoring the order, nor were significant interaction effects obtained.

Taken together, the effect of HL on the Secondary task performance at baseline driving was more skipped letters and fewer correctly recalled letters (especially when ignoring the order of letters). There was no significant effect of Task difficulty. 


\section{Secondary task at critical events (2 levels)}

No significant differences as a function of Gender and Hearing Status emerged from the subjective ratings (the adapted SART) of critical events.

There was a significant main effect of Task difficulty, such that there were fewer correct letters in the higher Difficulty Level, $F(1,44)=10.30, p=0.001, \eta_{\mathrm{p}}{ }^{2}=0.16$. There was also a significant main effect of Gender, such that men had more correct letters, $F(1,44)=9.15, p$ $=0.004, \eta_{\mathrm{p}}{ }^{2}=0.08$. There was also a significant interaction effect between Hearing Status and Task difficulty, such that Task difficulty affected the number of correct more for the individuals with $\mathrm{HL}, F(1,44)=3.66, p<0.05, \eta_{\mathrm{p}}{ }^{2}=0.02$. No significant main effect of Hearing Status and no additional interaction effects emerged.

There was a significant main effect of Task difficulty, such that more letters were skipped at the higher-Difficulty Level, $F(1,44)=9.10, p=0.003, \eta_{\mathrm{p}}{ }^{2}=0.09$. There was a significant effect of Hearing Status, such that participants with HL skipped more letters, $F(1,44)=5.54$, $p=0.02, \eta_{\mathrm{p}}{ }^{2}=0.10$. No significant main effect of Gender and no significant interaction effects emerged.

The number of correct recalled letters, regardless of the order, showed a significant main effect of Gender, such that men correctly recalled more letters, $F(1,44)=7.24, p=0.01, \eta_{\mathrm{p}}{ }^{2}$ $=0.13$. There was also a significant main effect of Hearing Status, such that participants with $\mathrm{NH}$ had more correct letters, $F(1,44)=4.94, p=0.03, \eta_{\mathrm{p}}{ }^{2}=0.09$. A significant effect of Task difficulty emerged, such that the lower Difficulty Level resulted in more correct letters, $F(1$, $44)=5.65, p=0.02, \eta_{\mathrm{p}}{ }^{2}=0.10$. No significant interaction effects were found.

In summary, participants with HL skipped more letters and recalled less correct letters during critical events compared to NH participants. Participants with HL were also more affected by the difficulty of task reflected by the correct serial order of the repeated letters.

\section{Secondary task at the Parked car event (1 level)}

All participants performed the task at the Parked car event and all were exposed with the more demanding Difficulty Level. No significant differences were found in terms of number of correctly recalled letters, skipped letters or correct letters disregarding order for Hearing Status or Gender, and neither was there an interaction effect between Hearing Status and 
Gender. Thus, there was no effect of HL or Gender on the Secondary task at the Parked car event.

\section{Discussion}

The aim of this study was to compare the effects of a secondary cognitive workload in individuals with and without HL, respectively, in driving situations with various complexities. During three different driving conditions, a Secondary task was present, with two levels of difficulty in terms of load on the phonological loop. Furthermore, a tactile alerting signal, given by a vibration in the seat, was evaluated in terms of effectiveness when calling for driver attention. Table 5 displays a summary of how the expectations were supported or not by the results.

\section{Table 5 about here}

The expectations regarding the more cautious driving behavior in the HL group was supported by the lower performance on the Secondary task, suggesting lower motivation to shift focus from the driving task. Also the lower driving speed for the HL group compared to the $\mathrm{NH}$ group, at the Parked car event, is in line with this assumption. However, more cautious driving behavior was not found for the baseline driving condition.

In line with the assumption that the effect of increasing complexity in the driving situations would be larger on the HL group is the decreased driving speed for the HL group around the Parked car event. This assumption is also supported by the lower driving speed for the HL group while performing the Secondary task and by the significant interaction effect of Task difficulty and Hearing Status at critical events, which did not appear at baseline driving. Additionally, the Mean TLCmin decreased for the HL group during the Secondary task and too small TLCmin values are strong indicators of reduced lateral control (Johansson, 2004). However, a Mean TLCmin of 5 or 6 seconds is long and indicates that both groups drove somewhat in the middle of the lane with sufficient safety margins. A longer braking distance at the Parked car event was also expected and could be suggested by visual inspection of the mean driving speed shown in Figure 1, however, no significant difference appeared in the analysis. All participants performed the Secondary task at the Parked car event, and there was no effect of Hearing Status or gender on the Secondary task performance in this specific situation. Thus, the assumption of a decrease in Secondary task performance in this specific situation due to HL was not supported. According to previous research this implies that for 
maintaining an acceptable difficulty range in this particular situation, neglecting the Secondary task was not necessary for any of the groups (cf. Fuller, 2005).

We assumed that the phonological similarity effect would affect participants with HL more. This was supported by the significant interaction effect of task difficulty and Hearing Status, which emerged for the number of correctly and orderly recalled letters at the critical events. At critical events, and for the less demanding level, the effect of HL on Secondary task performance was in line with that at the baseline driving (more skipped letters and fewer correct letters). However, at the more demanding level no significant main effect of HL was found. There may have been a floor effect at this more demanding level due to that all participants were interrupted in the middle of a non-forced task, since they were put in a near collision situation.

The differences related to HL during baseline driving in the simulator were mainly associated with the experience of the driving simulator. $\mathrm{NH}$ drivers experienced the simulator vibrations as more realistic. This could have had an effect on the driving speed which was significantly lower in the HL group. Female drivers with HL had the lowest ratings on vibration realism and also experienced the highest degree of discomfort in the simulator. These measures could be connected, such that lower experience of vibration realism leads to more motion sickness. Possibly both these measures could also be affected by HL, however significance appeared only for female drivers in the HL group. Further, for the female participants, the difference between PTA best ear and PTA worst ear was larger. If the asymmetry is not compensated, this can lead to balance problems or motion sickness (Johnson, 1999). Other studies have shown that women are more sensitive to simulator sickness (see Henriksson, 2009). The simulator sickness could also have a connection with the Secondary task, which was experienced as more difficult by the women.

An abstraction of the tested assumptions suggests that, when the driving complexity exceeds baseline driving, a more cautious driving behavior in the HL group compared to the NH group appears. This more cautious driving behavior could be a way of compensating for or adapting to the greater effect on the HL group when driving complexity increased. The driver behavior adaptation (or compensation) observed, decreased driving speed, and disregard of the Secondary task conforms well with previous research (Fuller, 2005; Lewis-Evans, 2011). The finding that the phonological similarity effect only affects the HL group more in critical situations also points at the effect of driving complexity. A way of compensating for this could be to invest less effort in the Secondary task, especially when the task difficulty 
increases. HL had no effect on the subjective ratings of how critical the critical events were experienced (as measured with the adapted SART). This is in line with Fuller (2005), such that drivers automatically try to maintain a constant level of workload.

In general the participants were positive to the way of announcing the Secondary task by seat vibration. No effect of HL or gender emerged, which suggests this modality as suitable regardless of gender and Hearing Status.

\section{Conclusions}

From these results we can conclude that HL had no effect on driving behavior at baseline driving, where no critical events occurred. However, in general, participants with HL had lower Secondary task performance, which was reflected in fewer correctly recalled letters, suggesting they are less willing to spend effort to perform the Secondary task.

The effect of increasing complexity in the driving situations was higher on drivers with HL. This was demonstrated by lower driving speed for the HL group at the Secondary task, lower driving speed for the HL group at the Parked car event, and a stronger effect of phonological similarity (i.e., Difficulty Level in the Secondary task) for the HL group at critical events.

We can also conclude that seat vibration is a feasible modality for announcing information to drivers, regardless of whether or not they have HL. The results from this study support the Task Capability Interface Model (Fuller, 2000) and suggests further research on design of driver support systems accessible for drivers with hearing loss. 


\section{References}

Andersson, U. (2002). Deterioration of the phonological processing skills in adults with an acquired severe hearing loss. European Journal of Cognitive Psychology, 14.

Arlinger, S. (2007). Nordisk Lärobok i Audiologi. Nordic Textbook of Audiology. In. Bromma: C-A Tegnér AB.

Baddeley, A., D. (2012). Working Memory: Theories, Models, and Controversies. Annu. Rev. Psychol., 2012, 1-29.

Baddeley, A. D. (1983). Working memory. Philos Trans R Soc, 302, 311-324.

Brookhuis, K. A., Waard, D. d., \& Fairclough, S. H. (2003). Criteria for driver impairment. Ergonomics, 46, 443 - 445.

Conrad, R., \& Hull, A. J. (1964). Information, acoustic confusion and memory span. British Journal of Psychology, 55, 429-437.

Endsley, M. R., \& Garland, D. J. (2000). Situation Awareness Analysis and Measurement. London: Lawrence Erlbaum Associates.

Engström, J., Johansson, E., Östlund, J.,. (2005). Effects of visual and cognitive load in real and simulated motorway driving. Transportation Research Part F, 8, 97-120.

Eysenck, M. W., \& Keane, M. T. (2010). Cognitive Psychology A Students Handbook (6 ed.): Psychology Press.

Fuller, R. (2000). The Task Capability Interface Model of the driving process. Recherche Transports Sécurité, 66, 47-59.

Fuller, R. (2005). Control and Effect: Motivational Aspects of Driver Decision Making. In L. Macchi, Re, C., Cacciabue, P.C. (Ed.), International Workshop on Modelling Driver Behaviour. Ispra, Italy: European Commision, Joint Research Centre.

Groeger, J. A. (2000). Understanding driving - Applying cognitive psychology to a complex everyday task: Psychology Press, Taylor and Francis, Hove, UK.

Henriksson, P. (2009). Simulator Sickness - Causes, Consequenses and Measures. VTI, Linköping, Sweden.

Hickson, L., Wood, J., Chaparro, A., Lacherez, P., \& Marszalek, R. (2010). Hearing Impairment Affects Older People's Ability to Drive in the Presence of Distracters. Journal of the American Geriatrics Society, 58, 1097-1103.

Ivers, R., Q., Mitchell, P., \& Cumming, R. (1999). Sensory Impairment and Driving: The Blue Mountains Eye Study. American Journal of Public Health, 89, 85-87.

Johansson, E., Engström, J., Cherri, C., Nodari, E., Toffetti, A., Schindhelm, R., Gelau, C. (2004). Review of existing techniques and metrics for IVIS and ADAS assessment.

Johnson, W. H., Sunahara, F.A, Landtholt, J.P. (1999). Importance of the vestibular system in visually induced nausea and self vection. J. Vest. Res., 9, 83-87.

Lewis-Evans, B., de Waard, D., Brookhuis, K. (2011). Speed Maintenance under Cognitive Load - Implications for Theories of Driver Behaviour. Accidents Analysis and Prevention, 43, 1497-1507. 
McCloskey, L. W., Koepsell, T. D., Wolf, M. E., \& Buchner, D. M. (1994). Motor-Vehicle Collision Injuries and Sensory Impairments of Older Drivers. Age and Ageing, 23, 267-273.

Michon, J. A. (1985). A critical view of driver behavior models: What do we know, what should we do? In L. A. Evans, Schwing, R.C. (Ed.), Human behavior and traffic safety. New York: Plenum.

Neisser, U. (1976). Cognition and reality: Principles and implications of cognitive psychology: W.H. Freeman and Company, New York.

Peters, B., \& Nilsson, L. (2006). Modelling the Driver in Control. In P. Cacciabue, C. (Ed.), Modelling Driver Behaviour in Automotive Environments- Critical Issues in Driver Interactions with Intelligent Transport Systems. London: Springer.

Picard, M., Girard, S. A., Courteau, M., Leroux, T., Larocque, R., Turcotte, F., Lavoie, M., \& Simard, M. (2008). Could driving safety be compromised by noise exposure at work and noise-induced hearing loss? Traffic Inj Prev, 9, 489-499.

Repovs, G., \& Baddeley, A., D. (2006). The Multicomponent Model of Working Memory: Explorations in Experimental Cognitive Psychology. Neuroscience, 139, 5-21.

Roth, T. N., Hanebuth, D., Probst, R. (2001). Prevalence of age-related hearing loss in Europe: a review. Eur Arch Otorhinolaryngol, 268, 1101-1107.

Schmolz, W. (1987). Die Bedeutung des Hoehrens im Verkehr. Polizei Verkehr Technik, 32, 379-380.

Sternberg, S. (1966). High-speed scanning in human memory. Science, 153, 652-654.

Thorslund, B., Peters, B., Lyxell, B., \& Lidestam, B. (2013). The Influence of Hearing Loss on Transport Safety and Mobility. European Transport Research Review, 5, 117-127.

Tsimhoni, O., Arbor, A (2003). Time-sharing of a visual in-vehicle task while driving: findings from the task occlusion method. The University of Michigan Transportation Research Institute (UMTRI), Michigan, USA. 


\section{Tables and Figures}

Table 1: Mean values in dB of PTA4 (mean of 500, 1000, 2000 and $4000 \mathrm{~Hz}$ ) in best and worst ear for men and women respectively in the $H L$ group.

\begin{tabular}{|l|ccc|ccc} 
& \multicolumn{3}{|c|}{ PTA4 Best ear } & \multicolumn{3}{c}{ PTA4 Worst ear } \\
& Mean & SD & Range & Mean & SD & Range \\
\hline Men $(n=13)$ & 45.6 & 22.6 & $6.2-\_97.5$ & 56.7 & 18.2 & $41.3 \ldots 107.5$ \\
Women $(n=11)$ & 35.8 & 17.7 & $20.0 \_62.5$ & 55.1 & 10.4 & $41.2 \ldots 73.7$
\end{tabular}

Table 2: Study design

\begin{tabular}{|c|c|c|c|c|c|}
\hline & \multirow{2}{*}{$\begin{array}{l}\text { Secondary } \\
\text { task- } \\
\text { difficulty }\end{array}$} & \multicolumn{2}{|c|}{$N H$} & \multicolumn{2}{|c|}{$H L$} \\
\hline & & Men & Women & Men & Women \\
\hline \multirow[t]{2}{*}{ Baseline } & Lower & 12 & 12 & 13 & 11 \\
\hline & Higher & 12 & 12 & 13 & 11 \\
\hline \multirow{2}{*}{$\begin{array}{l}\text { Critical } \\
\text { event }\end{array}$} & Lower & 12 & 12 & 13 & 11 \\
\hline & Higher & 12 & 12 & 13 & 11 \\
\hline Parked car & Higher & 12 & 12 & 13 & 11 \\
\hline
\end{tabular}

Table 3: Examples of the dependent variables; Number of correct (number of correct recalled letters in correct order), Number of skipped (number of skipped letters) and Number of correct recalled letters ignoring order. These variables were calculated for each participant and each task.

\begin{tabular}{cccccc}
\hline Letter sequence & Response & $\begin{array}{c}\text { Number } \\
\text { correct }\end{array}$ & $\begin{array}{c}\text { of } \\
\text { Number } \\
\text { skipped }\end{array}$ & of $\begin{array}{c}\text { Number of correct } \\
\text { ignoring order }\end{array}$ \\
\hline BDPT & BDTP & 2 & 0 & 4 \\
$R Q G H$ & RQ_H & 3 & 1 & 3 \\
\hline
\end{tabular}

Table 4: Experienced simulator sickness

\begin{tabular}{l|llcc}
\multirow{2}{*}{ Hearing Status } & Gender & $n$ & \multicolumn{2}{c}{ Mean Value } \\
& & & Mean & $S D$ \\
\hline \multirow{2}{*}{$N H$} & Male & 12 & 1.8 & 1.1 \\
& Female & 12 & 1.3 & 0.5 \\
& Male & 13 & 1.4 & 0.9 \\
& Female & 11 & 2.9 & 1.6
\end{tabular}


Table 5: Expectations and results

Expectations

Support

Yes

HL group will show a more cautious driving behavior.

Driving speed will be lower for HL group

No

Safety margins will be larger for HL group.

Motivation to perform the Secondary task will be $x$ lower in HL group.

The effect of increasing complexity in the driving situations will be larger on the HL group

Brake distance before the parked car will be

longer for the HL group.

Driving speed around the Parked car event will be lower for the HL group.

Secondary task performance will decrease more for the HL group.

For the HL group there will be a more apparent $x$

The phonological similarity effect wil strike harder on Participants with hearing loss.

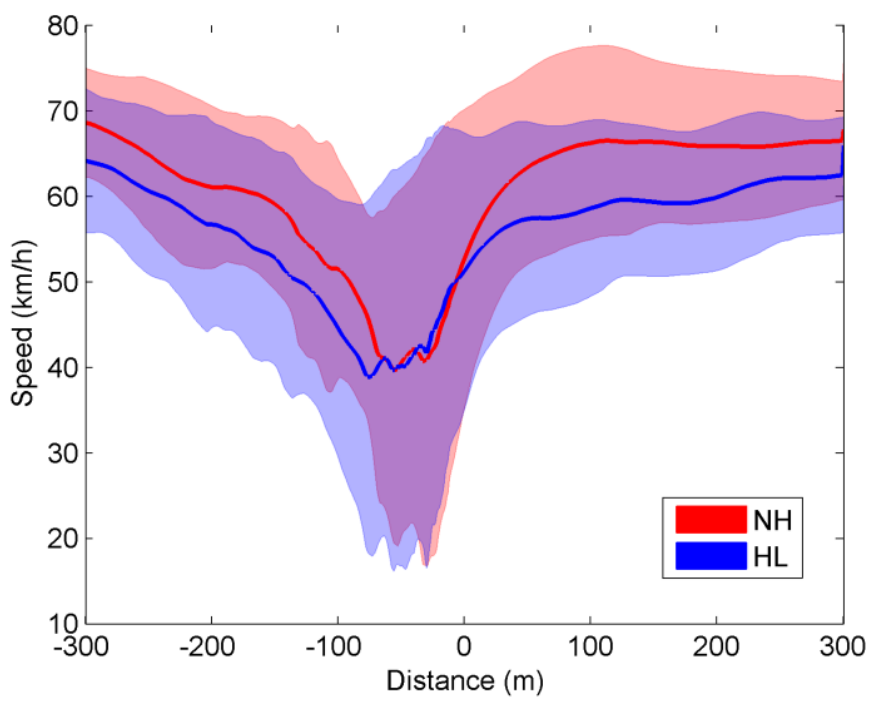

Figure 1: Mean driving speed for NH (red) and HL (blue) when passing a parked car. Shadow areas are standard deviations. Distance incudes 300 meters before the car to 300 meters after the car. 